\title{
MS06-03 | RNA Translocation Mechanism of Spliceosomal DEAH-box ATPases
}

Hamann, Florian (Georg-August-University Goettingen, Goettingen, GER); Schmitt, Andreas (Georg-August-University Goettingen, Goettingen, GER); Favretto, Filippo (Max Planck Institute for Biophysical Chemistry, Goettingen, GER); Zweckstetter, Markus (Max Planck Institute for Biophysical Chemistry, Goettingen, GER); Ficner, Ralf (Georg-August-University Goettingen, Goettingen, GER)

Spliceosomal DEAH-box ATPases were long thought to fulfill their tasks by direct unwinding of RNA duplexes. Recent studies, however, suggest that they rather function as ssRNA translocases and remodel the spliceosome from a distance by a mechanism called winching. Crystallographic studies on DEAH-box ATPases over the last decade have tried to shed light onto their motor function and the analyses of different ligand-bound states have been key to unravel the molecular dynamics. While early ADP-bound structures of Prp43 revealed the global architecture, later ATP- and RNA-bound structures unmasked insights into the RNA-loading and -binding mechanism and first domain movements could be described. More recently, adenosine nucleotide-free structures of Prp22 showed that DEAH-box ATPases are able to adopt more open conformations of the helicase core in the absence of ADP/ATP. By toggling between these closed and open conformations of the helicase core they are able to translocate along an ssRNA in $3^{\prime}-5^{\prime}$ direction with a step-size of one RNA nucleotide per hydrolyzed ATP. These dynamics additionally pose a particular challenge for other interaction partners, such as G-patch proteins that modulate the function of Prp43 and Prp2. The G-patch motif of the intrinsically disordered protein Spp2 is mostly unfolded in solution but its N-terminal part stably binds Prp2 via an amphipathic $\alpha$-helix, while the C-terminal part of this motif adopts two alternative conformations in different crystal structures. The increased conformational freedom of this region likely allows the G-patch to adapt to the different adenosine nucleotide-dependent conformations of the helicase core. 